University of Nebraska - Lincoln

DigitalCommons@University of Nebraska - Lincoln

Civil Engineering Faculty Publications

Civil Engineering

$6-2004$

\title{
Least-squares finite-element lattice Boltzmann method
}

Yusong Li

University of Nebraska - Lincoln, yli7@unl.edu

Eugene J. LeBoeuf

Vanderbilt University, Nashville, Tennessee

P. K. Basu

Vanderbilt University, Nashville, Tennessee

Follow this and additional works at: https://digitalcommons.unl.edu/civilengfacpub

Part of the Civil Engineering Commons

Li, Yusong; LeBoeuf, Eugene J.; and Basu, P. K., "Least-squares finite-element lattice Boltzmann method" (2004). Civil Engineering Faculty Publications. 10.

https://digitalcommons.unl.edu/civilengfacpub/10

This Article is brought to you for free and open access by the Civil Engineering at DigitalCommons@University of Nebraska - Lincoln. It has been accepted for inclusion in Civil Engineering Faculty Publications by an authorized administrator of DigitalCommons@University of Nebraska - Lincoln. 


\title{
Least-squares finite-element lattice Boltzmann method
}

\author{
Yusong Li, Eugene J. LeBoeuf,* and P. K. Basu \\ Department of Civil and Environmental Engineering, Vanderbilt University, Nashville, Tennessee 37325, USA
}

(Received 10 November 2003; published 2 June 2004)

\begin{abstract}
A new numerical model of the lattice Boltzmann method utilizing least-squares finite element in space and Crank-Nicolson method in time is presented. The new method is able to solve problem domains that contain complex or irregular geometric boundaries by using finite-element method's geometric flexibility and numerical stability, while employing efficient and accurate least-squares optimization. For the pure advection equation on a uniform mesh, the proposed method provides for fourth-order accuracy in space and second-order accuracy in time, with unconditional stability in the time domain. Accurate numerical results are presented through two-dimensional incompressible Poiseuille flow and Couette flow.
\end{abstract}

DOI: 10.1103/PhysRevE.69.065701

PACS number(s): $47.11 .+\mathrm{j}$

Application of the lattice Boltzmann method (LBM) to fluid flow suggests that it can be a powerful tool for solving complex fluid dynamics problems [1]. Traditional LBM, originating from lattice gas automata (LGA) where particles propagate from one site to another on a regular lattice, however, is restricted to a uniform grid. This limitation seriously affects the potential use of traditional LBM in many practical problems, e.g., flow in porous media, where complex pore geometries cannot be well represented by a uniform lattice [2]. While the coupling between discretization of velocity spaces and physical space is an essential part of LGA dynamics, it is not critical for LBM [3]. For example, interpolationsupplemented LBM can be implemented on an irregular rectangle $[4,5]$. Furthermore, LBM can be coupled with traditional numerical methods such as finite difference (FD), finite volume (FV), and finite element (FE) methods to extend the applicability of the LBM to irregular unstructured grids.

Based on Runge-Kutta time discretization and various spatial discretization schemes, Chen and co-workers [1,6] combined FD and LBM in a number of ways. The first central difference scheme was proposed by Cao et al. [6] in Cartesian coordinates, and was later extended to curvilinear coordinates with nonuniform grids [7]. A finite difference LBM (FD-LBM) scheme has been successfully applied in several aspects of fluid simulation [1], for example, for single-phase flow through three-dimensional digitized rock fractures under varied simulated confining pressures [8]. Amati et al. [9] were the first to propose a finite volume formulation of the LBM where a piecewise linear interpolation scheme was used to estimate the volume-averaged particle distribution in a nonuniform coarse lattice. Another volumetric formulation of LBM was developed by Chen [10], which can be applied to arbitrary meshes while achieving exact adherence to conservation laws and equilibrium conditions [10]. Peng et al. [11-13] proposed additional versions of the finite volume LBM (FV-LBM) for both triangular and rectangular elements, which appears to be flexible for both internal and external boundaries. The inherent geomet-

\footnotetext{
*Electronic address: eugene.j.leboeuf@vanderbilt.edu
}

ric flexibility and numerical stability of the finite-element method suggests that a finite-element LBM (FE-LBM) may be an appealing alternative to FV-LBM and FD-LBM.

While several works describe applications of FD and FV methods to LBM, few works have addressed FE-LBM. The popularity of FD-LBM and FV-LBM relative to FE-LBM derives from the nature of the classic Galerkin finite-element method. For equations with self-adjoint and positive-definite operators, the classic Galerkin finite-element method can lead to symmetric and positive-definite systems of linear algebraic equations. However, for non-self-adjoint equations, such as the lattice Boltzmann equation, classic Galerkin methods are often corrupted by spurious oscillations or wiggles [14]. Recently, Lee and Lin (2001) presented a characteristic Galerkin discrete Boltzmann equation (CGDBE) to overcome this problem by implementing a Taylor-Galerkin procedure for the discrete Boltzmann equation, which is, to the best of our knowledge, the only literature describing FELBM. This method, however, is limited by its conditional stability associated with the explicit expression of the convection term [15].

Least-squares finite-element (LSFE) method, on the other hand, was recently shown to be a robust and efficient way to solve non-self-adjoint equations where convection operators are of first order [14], always leading to symmetric, positivedefinite linear systems of equations, eliminating the need to use upwinding, staggered grids and operator splitting techniques [16]. Compared with Taylor-Galerkin-based FE methods, LSFE method possesses improved stability. Furthermore, for more complex systems, Taylor-Galerkin-based FE method may promote oscillations at discontinuities [14] or at solid-liquid interfaces. Those oscillations may be suppressed by artificially adding dissipation terms like those in "upwind" and "artificial viscosity" schemes, which, however, are dependent on the specific parameters of the problem. Employing a LSFE scheme to solve the lattice Boltzmann equation thus represents a promising approach to extend LBM to more practical and complex domains, while simultaneously benefitting from the finite-element method's superior stability and flexibility. It is in this light that we propose to implement a new FE-LBM, which utilizes LSFE in space and Crank-Nicolson scheme in time. 
Our starting point for illustrating this new method is the discrete lattice Boltzmann equation

$$
\frac{\partial f_{i}}{\partial t}+\vec{c}_{i} \cdot \vec{\nabla} f_{i}=\Omega_{i} \quad(i=1,2, \ldots N),
$$

where $f_{i}$ represents the particle velocity distribution function, $\vec{c}_{i}$ is the velocity along the $i$ th direction, $N$ is the number of different velocities in the model, and $\Omega_{i}$ denotes the collision operator which is commonly approximated by the Bhatnagar-Gross-Krook model [17],

$$
\Omega_{i}=-\frac{1}{\tau}\left(f_{i}-f_{i}^{e q}\right),
$$

where $\tau$ is the relaxation time and $f_{i}^{e q}$ is the local equilibrium given by

$$
f_{i}^{e q}=\rho \omega_{i}\left(1+\frac{\vec{u} \cdot \vec{c}_{i}}{c_{s}^{2}}+\frac{\left(\vec{u} \cdot \vec{c}_{i}\right)^{2}-c_{s}^{2} \vec{u}^{2}}{2 c_{s}^{4}}\right),
$$

in which $\omega_{i}$ is the weighting parameter for each velocity direction. The density per node $\rho$ and the macroscopic velocity $\vec{u}$ are defined by

$$
\rho=\sum_{i} f_{i} \quad \rho \vec{u}=\sum_{i} f_{i} c_{i} .
$$

Nine possible directional velocities are used in this study, where $\omega_{i}$ in Eq. (3) equals $4 / 9$ for $i=0,1 / 9$ for $i=1,2,3,4$, and $1 / 36$ for $i=5,6,7,8$. The nine velocities are defined as

$$
\vec{c}_{i}= \begin{cases}(0,0) & (i=0) \\ c_{s} \sqrt{2}\left(\cos \left[(i-5) \frac{\pi}{2}+\frac{\pi}{4}\right], \sin \left[(i-5) \frac{\pi}{2}+\frac{\pi}{4}\right]\right) & (1 \leqslant i \leqslant 4) \\ c_{s}\left(\cos \left[(i-1) \frac{\pi}{2}\right], \sin \left[(i-1) \frac{\pi}{2}\right]\right) & (5 \leqslant i \leqslant 8)\end{cases}
$$

Construction of the LSFE-LBM first considers application of the $\theta$ method to treat time-space approximations. Setting the time step $\Delta t=t^{n+1}-t^{n}$, and given $f_{i}^{n}$ for the previous time step, the solution $f_{i}^{n+1}$ for the current time step is determined from

$$
\begin{aligned}
& \frac{f_{i}^{n+1}-f_{i}^{n}}{\Delta t}+\theta \vec{c} \cdot \nabla f_{i}^{n+1}+(1-\theta) \vec{c} \cdot \nabla f_{i}^{n} \\
& \quad=\theta \Omega_{i}^{n+1}+(1-\theta) \Omega_{i}^{n} .
\end{aligned}
$$

Here, $\theta=1 / 2$ corresponds to the Crank-Nicolson scheme, providing for second-order accuracy in time. Rearranging Eq. (6), a standard form for LSFE can be obtained:

$$
\begin{gathered}
c_{x} \frac{\partial f_{i}^{n+1}}{\partial x}+c_{y} \frac{\partial f_{i}^{n+1}}{\partial y}+A f_{i}^{n+1}=p_{i}, \\
A=\frac{2}{\Delta t}+\frac{1}{\tau}, \\
p_{i}=\left(\frac{2}{\Delta t}-\frac{1}{\tau}\right) f_{i}^{n}+\frac{1}{\tau}\left(f_{i}^{e q, n+1}+f_{i}^{e q, n}\right)-\left(c_{x} \frac{\partial f_{i}^{n+1}}{\partial x}+c_{y} \frac{\partial f_{i}^{n+1}}{\partial y}\right) .
\end{gathered}
$$

For brevity the operator $\boldsymbol{L}$ is used, and Eq. (7) can be written in the following form:

$$
L f^{n+1}=p .
$$

For finite-element analysis, the problem domain can first be subdivided into a set of finite elements, and then approximated by the solution $f_{h}^{e, n+1}$ in a finite subspace as

$$
f_{h}^{e, n+1}=\sum_{j=1}^{n} N_{j} f_{j}^{n+1},
$$

where $N_{j}$ denotes the element shape function, $n$ represents the number of nodes in an element, and $f_{j}$ is the nodal values at the $j$ th node. Introducing Eq. (9) into Eq. (8) for an element, we get

$$
E=L f_{h}^{e, n+1}-p_{h}^{e},
$$

where $E$ is the residual due to nodal approximation. The LSFE is based on the minimization of the squares of the residual for the subspace

$$
\begin{gathered}
\varphi\left(f^{n+1}\right)=\int_{\Omega_{e}} E^{2} d \Omega_{e}=\int_{\Omega_{e}}\left(L f_{h}^{e, n+1}-p_{h}^{e}\right)^{2} d \Omega_{e}, \\
\frac{d \varphi\left(f^{n+1}\right)}{d f_{\alpha}^{n+1}}=\int_{\Omega_{e}}\left(L N_{\alpha}\right)^{T}\left(L f_{h}^{e, n+1}-p_{h}^{e}\right) d \Omega_{e}=0, \\
\alpha=1,2, \ldots n
\end{gathered}
$$

where $\Omega_{e}$ is the domain of the $e$ th element, and the exponent $T$ denotes the transpose. For each element the following linear algebraic equations can be derived from Eq. (12): 


$$
K_{e} F_{e}^{n+1}=P_{e},
$$

where $F_{e}^{n+1}$ is the vector of nodal values at the current time step. $K_{e}$ is the elemental matrix given by

$$
K_{e}=\int_{\Omega_{e}} Q^{T} Q d \Omega_{e},
$$

where $Q=\left\{Q^{1}, Q^{2}, \ldots Q^{i}, \ldots Q^{n}\right\}$ is a $(1 \times n)$ vector, with $Q^{i}$ of the following form:

$$
Q^{i}=c_{x} \frac{\partial N_{i}}{\partial x}+c_{y} \frac{\partial N_{i}}{\partial y}+A N_{i}
$$

The element at vector $P_{e}$ in Eq. (13) is

$$
P_{e}=\int_{\Omega_{e}} Q^{T} p_{h}^{e} d \Omega_{e} .
$$

As presented in Eq. (7) $p_{h}^{e}$ is related to the previous time step $f_{i}^{n}$ and $f_{i}^{\text {eq, } n}$ values, and the current time step $f_{i}^{\text {eq, } n+1}$ value. An extrapolation is applied to express $f_{i}^{\text {eq }, n+1}$, as proposed by Mei and Shyy [7],

$$
f_{i}^{e q, n+1}=2 f_{i}^{e q, n}-f_{i}^{e q, n-1},
$$

where $f_{i}^{n}, f_{i}^{e q, n}$, and $f_{i}^{e q, n-1}$ can be approximated in the subspace similar to $f_{i}^{n+1}$, as

$$
f_{h}^{n}=\sum_{j=1}^{n} N_{j} f_{j}^{n}, \quad f_{h}^{e q, n}=\sum_{j=1}^{n} N_{j} f_{j}^{e q, n}, \quad f_{h}^{e q, n-1}=\sum_{j=1}^{n} N_{j} f_{j}^{e q, n-1} .
$$

Boundary conditions are implemented as proposed by Lee and Lin [15]: $f_{h}^{n+1}$ at the boundary are treated as part of the solution, and macroscopic boundary conditions are imposed through $f_{h}^{\mathrm{eq}}$.

Through this LSFE derivation process it is clear that the $K_{e}$ matrix in Eq. (13) is symmetric and positive definite. Preconditioned conjugate gradient methods can be used as efficient tools for solution. Furthermore, an element-byelement scheme may be developed, without the need to store the global matrix [14], effectively reducing memory storage requirements.

Implementing the same accuracy analysis procedure as presented by Lee and Lin [15] for the pure advection equation on uniform meshes, LSFE-LBM enjoys similar accuracy as the CGDBE method, i.e., fourth-order accuracy in space and second-order accuracy in time. Compared to the secondorder accuracy in space for FD-based LBM, it is clear that FE-based LBM greatly increases numerical accuracy. Application of von Neumann stability analysis to LSFE-LBM reveals unconditional stability with any Courant-FriedrichsLewy (CFL) number for the pure advection equation on a uniform mesh if $\theta$ in Eq. (6) is in the range of $(1 / 2,1)$. This unconditional stability, derived from the implicit nature of LSFE, provides a significant advantage over CGDBE, which is only conditionally stable due to its explicit treatment of the advection term. A comparison of accuracy and stability among LSFE-LBM, CGDBE, and FD-LBM is given in Table I. Stability analysis related to nonlinear equilibrium terms in
TABLE I. A comparison of accuracy and stability characteristics of FD-LBM, CGDBE, and LSFE-LBM for the pure advection equation on a uniform mesh. $\Delta x=$ element size. $\Delta t=$ time step. $e=$ discrete velocity in the characteristic direction [15].

\begin{tabular}{lcccc}
\hline \hline & FD-LBM & CGDBE & LSFE-LBM \\
\hline \multirow{3}{*}{ Accuracy } & Space & Second order & Fourth order & Fourth order \\
& Time & Second order & Second order & Second order \\
Stability $^{\mathrm{a}}$ & & Conditional/ & $\Delta t \leqslant \Delta x / 3|e|$ & Unconditional \\
& & Unconditional & & \\
\hline
\end{tabular}

${ }^{\mathrm{a}}$ Stability of FD-LBM is based upon the specific time discretization scheme used.

the lattice Boltzmann equation, however, suggests that the stability of the LSFE-LBM is dependent on a number of parameters, including time step, element size, wave number, and relaxation time. This complex dependence of the stability has also been reported by other studies for LBM on irregular grids $[3,18]$, and thus is not unique to LSFE-LBM.

Two simple examples, Poiseuille flow and Couette flow, are presented to demonstrate the validation of the proposed method. More complex examples will be presented in subsequent publications. An analytical solution to plan Poiseuille flow in a channel is provided by Eq. (19) [19]:

$$
v_{x}(y)=u_{\max }\left[1-\left(\frac{y}{H}\right)^{2}\right], \quad-H \leqslant y \leqslant H,
$$

where $x$ is the spatial longitudinal dimension, $y$ is the spatial transverse dimension, $u_{\max }$ is the maximum velocity at the parabolic velocity profile, and $H$ is the half-width of the channel. In our LSFE-LBM implementation, the initial flow velocity is zero, relaxation time, $\tau$, is 0.05 , particle density, $\rho$, is $1.0, u_{\max }$ is 1.0 , and $H$ is $5 / 6$. A periodic boundary condition is applied in the $x$ direction, and a body force $G$ $=2 \nu u_{\max } / H^{2}$ is applied in the $x$ direction to initiate the flow, where $\nu$ is the viscosity following the relationship $\nu=\tau / 3$. This system possesses a Reynolds number $\left(R_{e}=u_{\max } 2 H / \nu\right)$ of 10 , and a Mach number $\left(\mathrm{Ma}=u_{\max } / c_{s}\right)$ of 0.173 . Results presented in Fig. 1 illustrate that LSFE-LBM achieves close agreement with the analytical solution.

The second application of unsteady Couette flow is used to evaluate the temporal accuracy of LSFE-LBM. Here, the top plate is moving along the $x$ direction at a constant velocity, $u_{\max }$, while the bottom plate remains stationary. The analytical solution for Couette flow is [19]

$$
u(y, t)=u_{\max } \frac{u}{D}+\sum_{i=1}^{\infty} \frac{2 u_{\max }(-1)^{i}}{\lambda_{i} D} e^{-1 / \lambda_{i}^{2} t} \sin \lambda_{i} y, \quad 0 \leqslant y \leqslant D
$$

where $\lambda_{i}=i \pi / D, \quad m=1,2,3 \ldots$

A periodic boundary condition is applied in the $x$ direction, and the Reynolds number $\left(R_{e}=u_{\max } D / \nu\right)$ is again set equal to 10 , where $D$ represents the width of the channel. The time step is 0.03 , relaxation time, $\tau$, is 0.05 , particle 


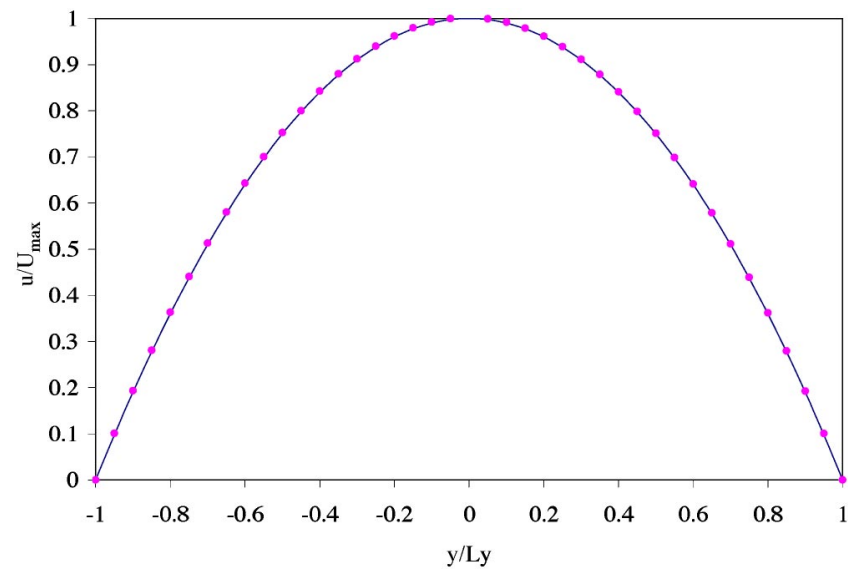

FIG. 1. Comparison of LSFE-LBM solution (points) and analytical solution (line) for normalized velocity profile for Poiseuille flow. In the LSFE-LBM, the relaxation time, $\tau$, is 0.05 , and particle density, $\rho$, is 1.0 , the maximum velocity, $u_{\max }$, is 0.1 , and the halfwidth of the channel, $H$, is $5 / 6$.

density is $1.0, u_{\max }$ is 0.1 , and $D$ is $5 / 3$. A comparison of the numerical results and the analytical solution is shown in Fig. 2.

Although the examples presented here are fairly simplistic in nature, they serve to demonstrate the successful coupling of LSFE and LBM, and its application to steady and unsteady incompressible flow. The geometric flexibility and numerical stability of finite-element methods inherent in LSFELBM suggest that this method is very flexible and can be applied to domains possessing complex boundary geometries using unstructured meshes with increased numerical accuracy and stability. LBM provides for increased computational efficiency relative to traditional computational fluid dynamics (CFD) methods employing the Navier-Stokes equation, especially in cases with increased geometric complexity. Al-

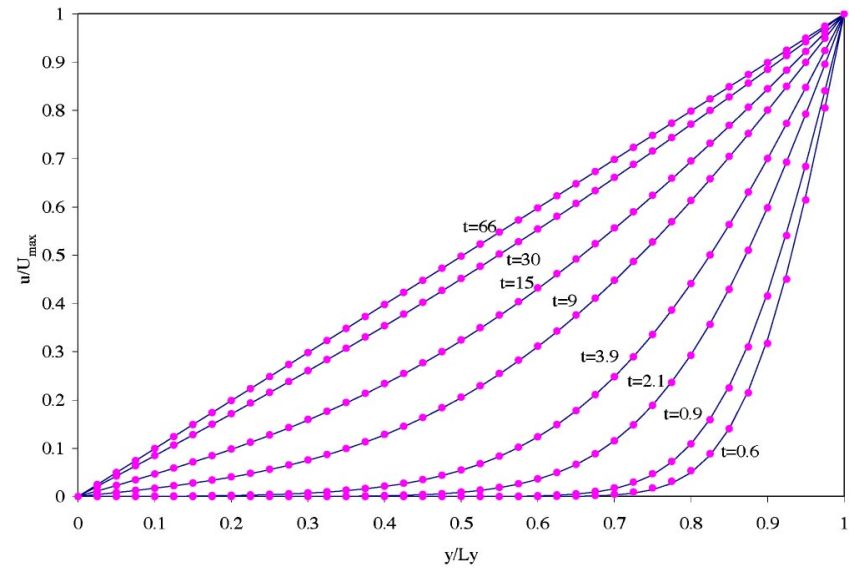

FIG. 2. Comparison of LSFE-LBM solution and analytical solution for Couette flow. The points represent the LSFE-LBM solution, while the lines denote the analytical solution. The time step is 0.03 , and relaxation time, $\tau$, is 0.05 , the maximum velocity, $u_{\max }$, is 0.1 , and the width of the channel, $D$, is $5 / 3$.

though the inherent computational requirements of FE-based LBM leads to reduced computational efficiency relative to the classic LBM, it is worthy to note that the use of unstructured mesh in LSFE-LBM is expected to require fewer grid points than methods using structured mesh, potentially offsetting the increased computational requirements of FE. A detailed discussion and comparison of computational efficiencies for LSFE-LBM, classical LBM, and other traditional CFD methods will be presented in a subsequent paper.

This material is based upon work supported by the National Science Foundation under Grant No. 0088912. Any opinions, findings, and conclusions or recommendations expressed in this material are those of the authors and do not necessarily reflect the views of the National Science Foundation.
[1] S. Chen and G. D. Doolen, Annu. Rev. Fluid Mech. 30, 329 (1998).

[2] S. Succi, G. Amati, and R. Benzi, J. Stat. Phys. 81, 5 (1995).

[3] J. D. Sterling and S. Chen, J. Comput. Phys. 123, 196 (1996).

[4] X. He, L.-S. Luo, and M. Dembo, J. Comput. Phys. 129, 357 (1996)

[5] X. He and G. D. Doolen, J. Comput. Phys. 134, 306 (1997).

[6] N. Cao, S. Chen, S. Jin, and D. Martinez, Phys. Rev. E 55, R21 (1997).

[7] R. Mei and W. Shyy, J. Comput. Phys. 143, 426 (1998).

[8] I. Kim and W. B. Lindquist, Phys. Rev. E 67, 046708 (2003).

[9] G. Amati, S. Succi, and R. Benzi, Fluid Dyn. Res. 19, 289 (1997).

[10] H. Chen, Phys. Rev. E 58, 3955 (1998).

[11] G. Peng, H. Xi, and C. Duncan, Phys. Rev. E 58, R4124 (1998)
[12] G. Peng, H. Xi, and C. Duncan, Phys. Rev. E 59, 4675 (1999).

[13] H. Xi, G. Peng, and S.-H. Chou, Phys. Rev. E 59, 6202 (1999).

[14] B.-N. Jiang, The Least-Squares Finite Element Method: Theory and Applications in Computational Fluid Dynamics and Electromagnetics (Springer, New York, 1998).

[15] T. Lee and C.-L. Lin, J. Comput. Phys. 171 (2001).

[16] X. Ding, "Large eddy simulation of turbulent transport processes by a least-squares finite element method," $\mathrm{Ph} . \mathrm{D}$. dissertation, University of Kentucky, Lexington, KY, 1999.

[17] P. Bhatnagar, E. P. Bross, and M. K. Krook, Phys. Rev. 94, 511 (1954).

[18] Z. Guo and T. S. Zhao, Phys. Rev. E 67, 066709 (2003).

[19] W. M. Deen, Analysis of Transport Phenomena (Oxford University Press, New York, 1998). 